\title{
Wissenstransfer im Kontext der Schweizer Curriculumdiskussionen der 1970er-Jahre
}

\section{Lukas Höhener und Lucien Criblez}

Der vorliegende Beitrag analysiert drei zentrale Transfermechanismen im Kontext der Schweizer Curriculumdiskussionen der 1970er-Jahre am Beispiel der Freiburger Arbeitsgruppe für Lehrplanforschung (FAL): den Transfer amerikanischer Forschungsergebnisse in Schweizer Kontexte der Curriculumreform, den Transfer von wissenschaftlichem Wissen über den Seitenwechsel des Personals von der Universität in die Bildungspraxis und die Bildungspolitik sowie den Wissenstransfer durch Politikberatung. Es werden Strategien und Motive der involvierten Akteure beleuchtet und Reformprozesse analysiert, die insgesamt aufzeigen, dass die FAL wesentlichen Anteil an der Verwissenschaftlichung der Lehrplanarbeit in der Schweiz der 1970 er-Jahre hatte.

Mit der Freiburger Arbeitsgruppe für Lehrplanforschung (FAL) formierte sich am Pädagogischen Institut der Universität Freiburg in der Schweiz 1968 ein Akteur, der sich im Feld der Curriculumforschung, -theorie und -entwicklung prominent positionierte, der aber dank der Themenkonjunktur in der Erziehungswissenschaft und dank des entsprechenden Beratungsbedarfs in der Bildungspolitik auch in der Bildungsforschung schnell an Bedeutung gewann. Die Curriculumforschung hatte zu Beginn des 20. Jahrhunderts in den USA mit John Dewey und der "progressive education» ihren Anfang genommen (Kliebard, 1987) und in der Mitte des 20. Jahrhunderts mit Ralph W. Tyler's «Rationale» (Tyler, 1949) und Benjamin S. Blooms Arbeiten zur Lernzieltaxonomie (Bloom et al., 1956/1972; Bloom \& Krathwohl, 1964) eine theoretische Weiterentwicklung erfahren (Hameyer, 1983). Sie beeinflusste die Schulreformdiskussionen anschliessend nicht nur in den USA, sondern auch in Europa und insbesondere im deutschsprachigen Raum. ${ }^{1}$ Allerdings wurde die Curriculumdiskussion hier erst in den späten 1960er-Jahren durch Saul B. Robinsohn's «Bildungsreform als Revision des Curriculums» (1967) initiiert. Robinsohn nahm darin die amerikanische Diskussion auf und adaptierte entsprechende Reformpostulate an die Situation in Deutschland. Wegen des grossen Bedarfs an wissenschaftlicher Expertise im Kontext der Bildungsexpansion wurde das 
Büchlein stark rezipiert und leitete in Deutschland einen Aufschwung der Forschung zu Curriculumthemen ein (Göldi, 2011; Hameyer, 1983).

Die in der Schweiz vorrangig durch die Forschergruppe an der Universität Freiburg vertretenen Konzepte, die sich generell der Curriculumtheorie und -forschung zuordnen lassen, können vor diesem Hintergrund nicht auf genuin schweizerische Ideen zurückgeführt werden, sondern sie verweisen auf Wissensund Wissenschaftstransfers zwischen verschiedenen Nationen, Expertengruppen, wissenschaftlichen Disziplinen und Institutionen. Die blosse Beschreibung solcher Transferprozesse als Übernahme von Ideen und Konzepten greift für das Verständnis der Herkunft der Curriculumtheorie sowie deren Rezeption und weiteren Verbreitung aber ebenso zu kurz (Ash, 2006) wie einfache Erklärungen der Nachahmung in handlungsunsicheren und zukunftsoffenen Situationen im Sinne von mimetischen Isomophieprozessen, wie sie etwa von neoinstitutionalistischen Theorien und Konzepten verwendet werden (Di Maggio \& Powell, 1983/2009). Vielmehr stellt sich die Frage, inwiefern der Wissenstransfer mit der Transformation von Wissen, Theorien, Konzepten und Modellen verbunden war, also eine Passung der Wissensbestände an neue kontextuelle Faktoren erfolgte, welche Mechanismen den Transfer begünstigten und welche dahinter stehenden Strategien und Motive der Akteure welche Wirkungen erzielten (Joyeux-Prunel, 2002).

An dieser Stelle setzt der folgende Beitrag an. Am Beispiel der Freiburger Arbeitsgruppe für Lehrplanforschung (FAL), die erstens die angelsächsische Curriculumtheorie rezipierte und auf dieser Grundlage entsprechende Modelle und Konzepte entwickelte, zweitens empirische Forschung im Rahmen der Curriculumentwicklung in der Schweiz initiierte, drittens dafür sorgte, dass die Kantone die Curriculumtheorie zur Leitvorstellung ihrer Lehrplanrevisionen machten, viertens sich später in der Aus- und Weiterbildung von Lehrerinnen und Lehrern für die Implementation dieser Leitvorstellung engagierte und fünftens letztlich auch die Lehrplanentwicklung und -implementation in einzelnen Kantonen begleitete, analysiert er drei zentrale Wissenstransfermechanismen: erstens den Transfer von amerikanischen (psychologischen) Theorien und Konzepten in die Schweizer Curriculumdiskussion, zweitens den Transfer von wissenschaftlichem Wissen aus der Universität in die Bildungsverwaltung und die Lehrerinnen- und Lehrerbildung durch den Seitenwechsel des Personals sowie drittens den Transfer in die Bildungspolitik durch Politikberatung. ${ }^{2}$ Zugleich wird aufgezeigt, welche Faktoren und Bedingungen den (Wissens-)Transfer unterstützten und förderten. Am Beispiel der Arbeiten der FAL kann auch gezeigt werden, wie die Lehrplanarbeit - ganz im Sinne der Robinsohn'schen Programmatik - verwissenschaftlicht wurde. Insbesondere durch die Beratungsmandate ist die FAL zudem ein gutes Beispiel für die Entstehung ,moderner' Politikberatung durch die Bildungsforschung. Es gilt deshalb abschliessend auch aufzuzeigen, wie durch die Dominanz eines einzelnen Akteurs wie der FAL, durch deren Politikberatungsfunktion und durch den 
Seitenwechsel der FAL-Mitglieder von der Bildungsforschung in die Bildungsverwaltung die Grenzen zwischen Bildungsforschung und Bildungspolitik subtil perforiert wurden.

\section{Die FAL und die Curriculumentwicklung}

In der Schweiz entwickelte sich die Freiburger Arbeitsgruppe für Lehrplanforschung in den 1970er-Jahren rasch zum wichtigsten Akteur der Curriculumtheorie und -forschung. Unter der strategischen Leitung von Karl Frey ${ }^{3}$ wurden einerseits die theoriebezogenen Forschungstätigkeiten vorangetrieben, woraus u.a. etliche Dissertationen der FAL-Mitglieder hervorgingen. Andererseits standen stets die Kontaktpflege zum Schulfeld und die Suche nach Kooperationen im ausseruniversitären Feld mit Lehrpersonen, Einzelschulen sowie kantonalen und interkantonalen Instanzen im Vordergrund (Eigenmann \& Schmid, 1978). Die Forschungstätigkeit der FAL wurde denn im Berufsfeld auch wahrgenommen und von der Bildungspolitik gefördert. Dies zeigt u.a. der Schlussbericht zur Enquête des Schweizerischen Wissenschaftsrates von 1970/1971, welcher die Curriculumforschung als vordringliches Forschungsbedürfnis im Bildungsbereich einstufte (Schweizerischer Wissenschaftsrat [SWR], 1973, S. 109). Die Tätigkeitsberichte der FAL verweisen auf Curriculumprojekte an den Primarschulen des Kantons Freiburg, den Weiterbildungsschulen Zug, der Oberstufe im Kanton Schwyz sowie auf Neukonzeptionen des Religionsunterrichts für die Deutschschweizer Volksschule oder auf die Zusammenarbeit in Lehrerbildungsfragen mit dem Pädagogischen Institut der Universität Zürich. Zudem verfassten die Mitglieder der FAL Gutachten zuhanden der von der Schweizerischen Konferenz der kantonalen Erziehungsdirektoren (EDK) eingesetzten Kommission «Lehrerbildung von morgen» (Müller et al., 1975) und führten Fortbildungsveranstaltungen durch, zum Beispiel für die "Groupe romand pour l'étude des techniques d'instruction» (GRETI) (Isenegger \& Santini, 1973).

Die grosse Aufmerksamkeit in den 1970er-Jahren für die Curriculumtheorie, -forschung- und -entwicklung fällt in eine Zeit des gesamtgesellschaftlichen Umbruchs und entsprechend intensiver Reformbestrebungen seit den späten 1950er-Jahren im Kontext der Bildungsexpansion (Criblez, 2012). Vor dem Hintergrund einer "Ausdifferenzierung der Bildungsoptionen" (Bosche, 2013, S. 13) und damit verbundenen Fragen der Chancengerechtigkeit standen zunächst die schulischen Strukturen im Zentrum der Reformdiskussionen. Als sich föderalistische Widerstände bei den strukturellen Koordinationsbemühungen der Kantone abzeichneten (Criblez, 2008; Manz, 2011), rückten die Lehrpläne als "Grundlage der Schulreform» (Aregger, Brunner \& Isenegger, 1972) und als Koordinationsinstrumente (Jenzer, Weiss \& Strittmatter, 1978) in den Fokus der Aufmerksamkeit. Nach einer Phase der Konzentration auf die ,äusseren' Schulreformen wandten sich die Bildungsreformer nun der ,inneren' 
Schulreform zu (Aregger, 1971a). Die Überarbeitung der kantonalen Lehrpläne wurde zu einem der wichtigsten Kernanliegen der kantonalen Bildungspolitik. Die zu Beginn der 1970er-Jahre innerhalb der kantonalen Bildungsverwaltungen geschaffenen Pädagogischen Arbeits- und Planungsstellen (Kussau \& Oertel, 2001; Rothen, 2016a, 2016b) übernahmen in der Regel die entsprechenden Entwicklungsaufgaben.

Lehrplanreformen erfolgten bis in die 1970er-Jahre pragmatisch und orientierten sich einerseits am wahrgenommenen Reformbedarf und an konstatierten gesellschaftlichen Problemlagen, zu deren Bearbeitung man sich von Bildungsreformen einen Beitrag versprach. Andererseits basierten Lehrpläne wenig reflektiert auf vorherrschenden gesellschaftlichen Wert- und Normvorstellungen sowie normativen Vorstellungen über, gute' Schulen, guten' Unterricht und ,gute' Bildung. Durch die Postulate und Ansätze der aufstrebenden Curriculumtheorie hielten nun neue, lernzielorientierte und theoriebasierte Leitvorstellungen Einzug. Sie versprachen einen «möglichst rational überprüfbaren und objektivierbaren Prozess» der Lernzielformulierung (Nicklas, 1972, S. 12). Auf diese Weise schienen sowohl das Problem der anwachsenden und kaum mehr begrenzbaren Auflistungen in den bisherigen Stofflehrplänen als auch das Problem der inhaltlichen Festlegungen, die als historisch zufällig, ja als beliebig wahrgenommen wurden, bearbeitbar zu werden (Künzli, 2002). Die curriculare Dekade der 1980er-Jahre war deshalb geprägt von intensiver Lehrplanarbeit, die in der deutschsprachigen Schweiz flächendeckend zur Einführung von lernzielorientierten Lehrplänen führte (Künzli, 2002/2006). ${ }^{4}$

\section{Transfer und Transformation in drei Akten}

In dreierlei Hinsicht stehen im Folgenden die Transfer- und Transformationsleistungen der Freiburger Arbeitsgruppe im Fokus. Zunächst wird gefragt, wie die Mitglieder der FAL durch ihre Forschungstätigkeit die Nutzung curriculumtheoretischer Konzepte für die Lehrplanarbeit erst möglich machten. Mit Blick auf die Laufbahnen der FAL-Mitglieder stellt sich zweitens die Frage, inwieweit die Curriculumtheorie eine Weiterentwicklung oder Konsolidierung erfuhr; dies insbesondere, als das Personal der FAL die Berufslaufbahnen in Bildungsverwaltungen oder -institutionen fortsetzte. Schliesslich werden die Rolle des FAL-Personals und die damit verbundenen Funktionen ausgeleuchtet. Es wird aufgezeigt, wie die Mitglieder der FAL als Experten in beratender Funktion in die Reformprozesse einbezogen waren und wie sie dadurch auch die Implementation ,ihrer' Konzepte begleiteten 5 .

\section{Die Rezeptions- und Transformationsleistung}

Mitte der 1960er-Jahre begann sich das Pädagogische Institut der Universität Freiburg thematisch auf Fragen rund um Lehrpläne und Curricula zu fokus- 
sieren und intensivierte seine diesbezüglichen Forschungstätigkeiten, bis sich aus diesen Aktivitäten um 1968 die FAL konstituierte. Etwa zur selben Zeit wurde die Reihe «Studien und Forschungsberichte aus dem Pädagogischen Institut der Universität Freiburg/Schweiz» lanciert. Bis Mitte der 1970er-Jahre erschienen über ein Dutzend Bände, etwa zu Lehrplänen der Real-, Sekundar- und Bezirksschulen der Schweiz (Frey, 1968b), zum Bildungsauftrag der Realschule (Frey, 1968a), zum Ausbildungsgang der Lehrpersonen (Frey, 1969a) oder später zu Kriterien der Curriculumkonstruktion (Frey, Lattmann \& Aebli, 1971). Daneben sind weitere Zeitschriftenartikel, Sammelbände und Monographien der FAL-Mitglieder zu ihren je spezifischen Forschungsbereichen entstanden. Die kursorische Analyse dieser, gleichsam als Anfänge der Schweizer Curriculumforschung zu bezeichnenden Publikationen, zeigt etliche Anknüpfungspunkte zu Diskussionen in den USA und im übrigen deutschen Sprachraum auf, die im Folgenden im Zentrum des Interesses stehen.

«Die Lehrplananalyse dient dem Aufbau der künftigen Curricula bzw. [von] wissenschaftlich fundierten Lehrpläne[n]», schrieb Karl Frey als damaliger Leiter der FAL im «International Review of Education» (Frey, 1969b, S. 24). An diesem Zitat wird deutlich: Die Begriffe «Lehrplan» und «Curriculum» werden neu mit dem Anspruch auf Wissenschaftlichkeit verbunden, aber nicht wirklich voneinander abgegrenzt. ${ }^{6}$ Das Ziel eines Symposions über Curriculumforschung an der Universität Freiburg (2.-6. Oktober 1973) bestand deshalb darin, den Curriculumbegriff zu klären.

Es wurde unterschieden zwischen dem «Curriculum als Dokument, als kodifizierte Form von Bildungsabsichten» einerseits und dem «Curriculum als Insgesamt von (geplanten) Aktivitäten, welche Unterricht beeinflussen», andererseits (Isenegger, 1975, S. 10). Im deutschen Sprachraum habe man sich vornehmlich der ersten Definition bedient. Aus dieser Sicht sei das Curriculum als Lehrplan, Lernzielkatalog oder Materialsystem zu verstehen, während die zweite, weitläufigere Definition im angelsächsischen Sprachraum oder in OECD-Kreisen gebräuchlich gewesen sei, ohne allerdings weiter präzisiert zu werden. Die amerikanische Forschung prägte generell einen Curriculumbegriff mit einem viel weiteren Bedeutungsumfang als er damals mit dem deutschen Lehrplanbegriff abgedeckt wurde.

Die FAL verschrieb sich dem Ziel, wissenschaftlich fundierte Lehrplanarbeit leisten zu wollen. Das Grundkonzept dieser neuen Herangehensweise findet ihren Konnex in der «realistische[n] Wendung in der pädagogischen Forschung» (Roth, 1963). Und dazu hatte die angelsächsische Literatur bereits einiges anzubieten, was den Absichten und Vorstellungen der FAL entgegenkam. Der Blick in die ersten Publikationen von Frey und den ersten weiteren Mitgliedern der Arbeitsgruppe zeigt ein eindeutiges Bild. Die begrifflichen Einordnungen sind geprägt von Verweisen auf eine überschaubare Forschungsliteratur angelsächsischer Provenienz: Zu Schulreformen generell zitierte die FAL jeweils Goodlad (1964), zur Curriculumentwicklung wurde auf Taba (1962) verwiesen. 
Wenn die FAL Innovationen im Bildungsbereich ansprach, verwies sie auf Miles (1961) und beim Thema Taxonomie von Bildungszielen findet sich hauptsächlich der Verweis auf Bloom (Bloom et al., 1956/1972).

Insbesondere wenn sich die Schweizer Curriculumentwicklung mit Fragen der «Auswahl und Anordnung von Bildungszielen, Unterrichtsthemen, Lernverfahren und Evaluationsmethoden» beschäftigte, werden Bezüge zur amerikanischen Forschung deutlich (Frey, 1970a, S. 50). Die Absicht der hiesigen Konstrukteure von Curricula bestand unter anderem darin, überprüfbare Lernziele zu formulieren, wozu sie Kriterien, Bezugspunkte und Klassifikationsschemata (Taxonomien; vgl. Bloom et al., 1956/1972) benötigten. Dazu griffen sie auf Modelle aus der einschlägigen Literatur der Psychologie aus den USA zurück und entwickelten sie weiter (Santini, 1983). So finden sich fortwährend Bezüge zu lernpsychologischen Konzepten und Modellen von Gagné (1965/1969), Bruner (1966) und Ausubel (1968). Zur Erfassung von Persönlichkeitsmerkmalen knüpfte die FAL jeweils an Guilford (1959/1964) an. Diese Konzepte konnten aber nicht bloss rezipiert werden, sondern sie mussten im neuen bildungspolitischen Kontext eingeordnet und weiterentwickelt werden. Die Rezeption im Kontext der Schweizer Reformbemühungen war deshalb zwingend mit Transformationen verbunden. Erst diese Transformationsleistungen der FAL führten dazu, dass die Konzepte der Curriculumtheorie und -entwicklung in den Kantonen auch zur Grundlage der Lehrplanreformen werden konnten.

Neben diesen modell- und theoriebildenden Aktivitäten stand die FAL in intensivem Austausch mit dem pädagogischen Praxisfeld, und zwar sowohl durch die Kommunikation der Resultate empirischer Untersuchungen und Erhebungen als auch durch konkrete, lehrplanbezogene Projektarbeiten in mehreren Kantonen. In drei gross angelegten Untersuchungen erarbeitete sich die FAL zunächst einen Überblick über die Lehrpläne der Real-, Sekundar- und Bezirksschulen (Sekundarstufe I) in der Schweiz (Frey, 1968b), dann analysierte sie die Strukturen der schweizerischen Volksschullehrerbildung (Frey, 1969a) und untersuchte schliesslich die "Ausbildung und Anstellung der Schweizer Gymnasiallehrer» (Horn, Morger, Regli \& Zumthurn,, 1969). Als generelle Erkenntnis resultierte aus den empirischen Forschungsarbeiten, dass sowohl hinsichtlich der Ausgestaltung der Lehrpläne als auch der Ausbildung der Lehrpersonen grosse Unterschiede zwischen den Kantonen bestanden. Die föderalistische Vielfalt wurde als Beliebigkeit interpretiert und auf dieser Grundlage Reformbedarf konstatiert. Von der EDK wurde die Untersuchung der Volkschullehrpläne überdies als Ausgangspunkt für die weitere Koordinationsplanung empfohlen (Frey, 1968b).

Im deutschsprachigen Teil des Kantons Freiburg prägte die FAL die Schulreform der Primarschule seit 1969 massgeblich, wobei unter ihrer wissenschaftlichen Begleitung der Schwerpunkt der Schulreform auf die Entwicklung der inneren Strukturen des Unterrichts gelegt wurde. Infolgedessen wurde einerseits die Revision der bisherigen Lehrpläne vorangetrieben, andererseits der 
Austausch und die Kooperation zwischen den verschiedenen Akteuren im Schulsystem intensiviert. Ferner wurde in diesem Projekt eine Aus- und Fortbildung für die am Curriculumprozess Beteiligten lanciert; damit wurden in erster Linie Lehrpersonen und Inspektoren angesprochen. Und schliesslich sollte der Aufbau eines sich selbst reformierenden Schulsystems vorangebracht werden (Isenegger, 1972). Aus dem Projekt ist nebst einem lernzielorientierten Lehrplan für die Primarschulen Deutschfreiburgs eine Vielzahl an Produkten hervorgegangen: u.a. Projektbeschriebe, Theoriemodelle, Teile der Habilitationsschrift von Karl Frey (1971), Curriculumentwürfe, Aufgabensammlungen für Übertrittsprüfungen oder ein lernzielbasiertes Hilfsinstrument für Lehrpersonen zur Unterrichtsvorbereitung und -verlaufsplanung.

Mit der Berufung von Karl Frey zunächst zum Professor und kurz darauf auch zum geschäftsleitenden Direktor des Leibniz-Instituts für die Pädagogik der Naturwissenschaften und Mathematik (IPN) in Kiel erlebte die Freiburger Curriculumforschung 1971 in gewissem Sinne einen Internationalisierungsschub. Das IPN verstand sich seinerzeit als "Curriculum-Entwicklungsinstitut» (Ringelband, 2016, S. 33). Einige Mitglieder der FAL folgten Karl Frey kurzzeitig ans IPN, und das IPN räumte einigen von ihnen in seinen Publikationen Platz ein. Kurt Aregger präsentierte beispielsweise in einem IPN-Band seine Ergebnisse zur Organisation der Curriculumentwicklung (1975) sowie in Kooperation mit Karl Frey "das Generative Leitsystem», ein Modell zur Integration von Theorie und Praxis in Curriculum-Projekten (Aregger \& Frey, 1975). Inwieweit aber die Erkenntnisse der Schweizer bzw. Freiburger Curriculumforschenden im internationalen Kontext rezipiert wurden, muss derzeit offen bleiben und Gegenstand weiterer Untersuchungen sein.

In der Schweizer Curriculumforschung hat die Freiburger Arbeitsgruppe für Lehrplanforschung in den späten 1960er- und den 1970er-Jahren sowohl das thematische Terrain besetzt als auch einen Teil der Reformagenda definiert. Sie folgte Robinsohns Appell nach curricularen Bildungsreformen und weitete in dieser Tradition die Lehrplanarbeit im Sinne angelsächsischer curriculum studies einerseits auf die explizite Formulierung von Bildungsabsichten und -zielen für den Unterricht, andererseits auf die wissenschaftliche Fundierung der Lehrplanentwicklung aus. Die FAL orientierte sich dabei insbesondere an der amerikanischen Literatur. Als grundlegendes Ziel der FAL kann zusammenfassend gelten, dass sie um die Schaffung wissenschaftlicher Grundlagen für die Konstruktion von rational begründeten und objektivierten Lehrplänen bemüht war, also Lehrplänen mit überprüfbaren Lernzielen, deren Konstruktion ein transparentes und theoretisch fundiertes Verfahren zugrunde liegt. Die FAL verband Rezeptions- mit adaptiven Transformationsleistungen und ermöglichte so der Bildungspolitik und -verwaltung in verschiedenen Kantonen, Lehrplan- und andere Reformprojekte neu wissenschaftlich zu fundieren.

Indem die Mitglieder der FAL schon Ende der 1960er-Jahre mit ihren Untersuchungen zur Situation der Lehrerinnen- und Lehrerbildung sowie zu den 
Lehrplänen den Status Quo analysierten und den bestehenden Reformbedarf aufzeigten, haben sie gleichsam ihr zu bearbeitendes Forschungs- und Entwicklungsfeld selber definiert. Dieses Forschungs- und Entwicklungsfeld bot für ihre curriculumspezifischen Modelle und Konzepte sowohl Anwendungs- als auch Weiterentwicklungsmöglichkeiten. Die wissenschaftliche Beteiligung an mehreren konkreten Projekten der Curriculumreform im Sinne von Entwicklungsaufträgen und Politikberatung war dann letztlich eine logische Folge des Agendasettings und der hegemonialen Besetzung des thematischen Feldes durch die FAL.

\section{Se itenwechsel}

Ein Blick auf die beruflichen Laufbahnen der FAL-Mitglieder wirft die Frage auf, inwiefern die vormals in Freiburg entstandenen Theorien und Konzepte eine Fortführung erfahren haben, nachdem einige der FAL-Mitglieder ihre Laufbahn in Verwaltungen oder Bildungsinstitutionen fortgesetzt und damit einen Seitenwechsel vollzogen hatten.

Im Zeitraum zwischen 1965 und 1975 kann eine intensive Phase des Ausbaus von Institutionen der Schweizer Bildungsforschung beobachtet werden, die bisweilen als «eigentliche Gründungswelle» bezeichnet wurde (Gretler, 2001, S. 312). Die Ursachen für diesen Institutionalisierungsschub können im damals herrschenden Reformdruck aufgrund gesellschaftlicher und wirtschaftlicher Modernisierungen und damit verbundenen bildungspolitischen Bedürfnissen verortet werden. Rationalitätssteigerung und Verwissenschaftlichung sollten die Bildungspolitik darin unterstützen, die Bildungsexpansion besser bewältigen zu können. Im Kanton Solothurn beispielsweise wurde die Notwendigkeit einer Pädagogischen Arbeitsstelle mit dem Dulliker Gesamtschulversuch begründet, der wissenschaftlich begleitet werden sollte (Jenzer, 1983). Die kantonale Bildungsverwaltung wurde deshalb mit einer Abteilung ergänzt, die wissenschaftliche Grundlagen beschaffen und diese für die Bildungsplanung konzeptionell nutzen sollte (Criblez, 2012). Ähnlich wurde die Schaffung des 1974 gegründeten Zentralschweizerischen Beratungsdienstes für Schulfragen (ZBS) in Luzern oder anderer Bildungsplanungsstellen begründet, die allerdings mit unterschiedlichen Aufgaben und Funktionen versehen wurden (Bain, Brun, Hexel \& Weiss, 2001; Rothen, 2016a, 2016b).

Ursprünglich strebten die Innerschweizer Kantone an, gemeinsam eine Reform der Sekundarstufe I7 in die Wege zu leiten. Die Erziehungsdirektoren gründeten deshalb auf Initiative einer interkantonalen Studiengruppe den ZBS zur Bearbeitung ihres ersten grossen gemeinsamen Projektes: «Lehrpläne für die Zentralschweiz» (Winiger, 2007). Die Gründung war auch durch die Einsicht motiviert, dass die Reform der Sekundarstufe I Jahre in Anspruch nehmen würde und dass sich die bisherige Reformpraxis mit Milizkommissionen überlebt habe. In der Folge wurden - vorerst auf fünf Jahre befristet - drei Stellen geschaffen und mit wissenschaftlichem Personal besetzt, um Lösungen für die anstehenden bildungspolitischen Probleme zu erarbeiten (Strittmatter, 1977). 
Die Leitung des ZBS übernahm im ersten Jahr Iwan Rickenbacher, ein ehemaliges Mitglied der FAL ${ }^{8}$. In dieser Zeit des Aufbaus galt es, einen Rahmenlehrplan für die unterschiedlichen Schulformen der Sekundarstufe I auszuarbeiten, die Selektion der Schülerinnen und Schüler am Übergang in die Sekundarstufe I neu zu konzipieren und zu reorganisieren sowie eine Auswertung der Vernehmlassung «Lehrerbildung von morgen» (Müller et al., 1975) vorzunehmen. Die Lehrplanarbeit, aus bildungspolitischer Sicht als Instrument der interkantonalen Koordination verstanden, zeichnete sich aus durch eine Erweiterung der Inhalte mit neuen Fächerbereichen und deren Reorganisation. Einzug hielten die Bereiche «Beruf und Wirtschaft» und die «Naturlehre». Für den Unterricht in diesen Fachbereichen fehlten allerdings entsprechende Lehrmittel.

Die Steigerung des Umfangs der neuen im Vergleich zu den bisherigen Lehrplänen und das Fehlen geeigneter Lehrmittel sind auf eine weitere lehrplanspezifische Neuerung zurückzuführen: die lernzielorientierte Ausrichtung der Lehrpläne (Winiger, 2007). Die Mitglieder der FAL hatten im Kanton Freiburg in ihrem ersten Projekt ihr Fachwissen zur Verfügung gestellt und entsprechende Erfahrungen gesammelt. Die Zentralschweizer Lehrplanreform kann durchaus als Fortsetzung der kantonalen Lehrplanreformen für einen andern geografischen Raum interpretiert werden - und sie wurde unter Anleitung derselben Akteure durchgeführt.

Allerdings fanden sowohl die Ergänzung der bestehenden Bildungsbehörden und -institutionen mit wissenschaftlichem Personal als auch die Einführung der Lernzielorientierung in den Lehrplänen unter kritischer Beobachtung statt (Rickenbacher, 1976; Winiger, 2007). Der ZBS verfolgte die Strategie, die lautesten Kritiker - und das waren allen voran die direkt betroffenen Lehrpersonen - mit in den Prozess der Lehrplanentwicklung einzubeziehen. Aus allen am Prozess beteiligten Kantonen und allen betroffenen Schulstufen wurden Vertreterinnen und Vertreter in den Arbeitsgruppen zur Mitarbeit aufgerufen. Der stärkere und frühzeitige Einbezug der Betroffenen in die Lehrplanarbeit war ein grundlegendes Postulat Robinsohns (vgl. oben), folgte gleichsam dem Implementationsansatz 9 der Freiburger Curriculumforscher (Aregger, 1971a) und kann als Transferstrategie verstanden werden.

Eine weitere Form des Seitenwechsels des wissenschaftlich qualifizierten Personals der FAL bestand in der Abkehr von der universitären Forschung zugunsten eines Engagements in der Lehrerinnen- und Lehrerbildung. Nach der einjährigen Aktivität beim ZBS engagierte sich Iwan Rickenbacher während über zehn Jahren als Direktor des Lehrerseminars des Kantons Schwyz in Rickenbach. Urs Peter Lattmann wurde 1972 zum Direktor der Aargauischen Lehramtsschule gewählt. Ihre Tätigkeiten fallen in eine Zeit der Systemüberprüfung der regional eingebetteten Mittelschulseminare. Als Reformanstoss innerhalb der Schweiz galten die in Freiburg durchgeführte Untersuchung unter dem Titel «Der Ausbildungsgang der Lehrer: eine Modellanalyse des Unterrichts in den 52 Lehrerbildungsanstalten zum Zwecke der Curriculumreform» (Frey, 1969a) 
und die in der Folge gebildete EDK-Expertenkommission zur «Lehrerbildung von morgen» (LEMO). Die Versuche zur Reform der Lehrerinnen- und Lehrerbildung in der Schweiz sind allerdings auch vor dem Hintergrund initiierter Akademisierungsprozesse der Lehrerinnen- und Lehrerbildung im Ausland zu verstehen (Criblez, 2010).

Das Anliegen, das Schulwesen über die Inhalte, und damit über die Curricula, zu koordinieren, bestand nicht nur im Volksschulbereich. Harmonisierungsdiskussionen wurden auch im Bereich der Lehrerinnen- und Lehrerbildung geführt, in der ersten Hälfte der 1970er-Jahre insbesondere in der LEMO-Kommission. Karl Frey und weitere FAL-Exponenten waren Mitglieder dieser Kommission ${ }^{10}$. Sie machten - ihrem Forschungsprogramm folgend - die Idee eines einheitlichen Curriculums für die Ausbildung der Lehrerinnen und Lehrer stark. Obwohl sich die Expertenkommission im Streit um das seminaristische und das maturitätsgebundene Ausbildungskonzept für Primarlehrerinnen und Primarlehrer nicht auf eine gemeinsame Struktur einigen konnte ${ }^{11}$, schlug sie ein Modellcurriculum für die Ausbildung von Primarlehrerinnen und Primarlehrern vor (Müller et al., 1975, S. 98ff.) - sicher auch ein Verdienst der FAL-Vertreter in der Kommission (Strittmatter, 2003).

Parallel zur Arbeit der LEMO-Kommission gestalteten Rickenbacher und Lattmann die Lehrerbildungsinstitutionen, denen sie vorstanden. Einerseits galt es, strukturelle Fragen zur (Neu-)Positionierung der Lehrerinnen- und Lehrerbildung zu bearbeiten, zumal ein wesentlicher Teil der Ausbildungsstätten zu Beginn der 1970er-Jahre noch als Mittelschulseminare konzipiert waren. Andererseits folgte die Neugestaltung dem «Anspruch einer wissenschaftlich fundierten und gleichzeitig auf die Praxis bezogenen Ausbildung» (Lattmann, 1976, S. 199). Die erfahrungsbasierte Tradition sollte - ganz im Sinne der Ambitionen und der grundlegenden Überzeugungen der FAL-Mitglieder - abgelöst werden. Die Überzeugung, dass Bildung geplant werden kann und geplant werden muss, hat zudem in den sogenannten «Netzplänen» eine Fortsetzung gefunden. «Netzpläne» sollten der Veranschaulichung des Wandels von Bildungsinstitutionen (Lattmann, 2015) dienen und können als gutes Beispiel für den wachsenden Einfluss der Sozialwissenschaften auf die Planungsprozesse im Bildungsbereich gelten (Bosche, 2016). Deutliche Bezüge zur Curriculumforschung wiesen auch die konzeptionellen Überlegungen zur tertiarisierten Lehrerinnen- und Lehrerbildung auf, etwa für die 1973 neu geschaffene Lehramtsschule und die Höhere Pädagogische Lehranstalt (HPL) im Kanton Aargau; entsprechende Erkenntnisse aus der Bildungs- und insbesondere der Curriculumforschung sollten in der Lehrerinnen- und Lehrerbildung berücksichtigt werden, so Lattmann als Gründungsdirektor beider Institutionen (1976, S. 199). Aus den Erfahrungen der von Bildungsexperten entworfenen und top-down verfügten Lehrplanprojekte in Deutschland habe man Lehren zu ziehen: Die Curriculumdiskussionen orientierten sich zwar nach wie vor an der Theorie, in die entsprechende Curriculumentwicklung würden aber auch die Lehrpersonen einbezogen. 
Was in der Curriculumcommunity einst als Handlungsforschungsprojekte begonnen hatte und etwa den Implementationsansatz zur praxisnahen oder schulnahen Curriculumentwicklung hervorgebracht hatte, fand in der Ausund Weiterbildung von Lehrerinnen und Lehrer später Anwendung mit einem Fokus auf Kooperation und Kommunikation zwischen Wissenschaft und Schulpraxis. Mitverantwortlich für den Vollzug dieses, Gesinnungswandels' waren die Seitenwechsel von promovierten Erziehungswissenschaftlern. Sie brachten neue Konzepte und Modelle aus ihrer bisherigen wissenschaftlichen Tätigkeit mit in die Lehrerinnen- und Lehrerbildung.

Das Beispiel der Einführung des Zentralschweizer Beratungsdienstes als Planungsinstanz der Innerschweizer Bildungsreformen hat veranschaulicht, wie das Erbe der Curriculumforschung der FAL eine Weiterführung in der bildungspolitischen Praxis gefunden hat. Die personellen Verflechtungen zwischen den Innerschweizer Bildungsinstitutionen und Vertretern der FAL könnten nun auf die traditionelle Verflechtung der katholischen Innerschweiz mit der katholischen Universität Freiburg zurückgeführt werden. Dass ein solches katholisches Netzwerk in den 1960er- und 1970er-Jahren noch bestand, kann kaum bestritten werden. Allerdings hatte insbesondere das zweite Vatikanische Konzil die traditionellen Verbindungen zwischen Schule und katholischer Kirche zu relativieren begonnen. Parallel zu diesem Prozess wurde die Wissenschaftsorientierung verstärkt. Und nicht nur das Beispiel der Aargauer Lehrerinnen- und Lehrerbildung (Criblez, Imlig, Montanaro-Batliner, Ruoss \& Völgyi, 2013; Metz, 2000) zeigt, dass die Transferprozesse durch Personal nicht einfach auf alte katholische Netzwerke zurückgeführt werden können. Denn FAL-Mitarbeitende haben auch wesentliche Funktionen in der Lehrplanreform des reformierten Kantons Bern übernommen.

\section{Beratungsleistungen}

Neben Theorie-, Modell- und Konzepttransfer sowie Transfer via Personal kann am Beispiel der FAL ein dritter Transferprozess verdeutlicht werden: der Transfer durch (Politik-)Beratung. Im folgenden Abschnitt stehen deshalb die vielseitigen wissenschaftlichen Beratungstätigkeiten der FAL in unterschiedlichen Praxisfeldern im Zentrum des Interesses. Gleichzeitig wird verdeutlicht, inwiefern die Komplexitätssteigerung in der Entwicklung von Lehrplänen und deren Implementation den Bedarf an qualifiziertem Personal und die Nachfrage nach Beratung steigerte.

"Wissenschaftliche Erkenntnisse der letzten Jahre zwingen uns zum weitgehenden Umdenken bei der Lehrplangestaltung. In zentralen Fächern wie Mathematik, Deutsch und Fremdsprachen werden völlig neue Wege eingeschlagen, die über kurz oder lang in den Stoffplänen ihren Niederschlag finden müssen» (Realschule Basel Stadt, 1975). Im Verlaufe der Basler Bildungsreform - ein weiteres Beispiel für die Aktivitäten der FAL - mit der ursprünglichen Absicht, die Koedukation an den Schulen der Sekundarstufe I einzuführen, wurden in 
der Überarbeitung der Lehrpläne zunehmend neue, wissenschaftliche Ansprüche formuliert. Schon in der Anfangsphase der mehrere Jahre dauernden Reform brachte sich das Institut für Unterrichtsfragen und Lehrerfortbildung (ULEF) unterstützend mit in die Diskussionen ein (Dunkel, 2014). Es beteiligte sich an der Ausarbeitung von Projektplänen und an der Reorganisation von Stundentafeln. Insbesondere als der Fokus auf die Ausarbeitung und Formulierung von Lernzielen zu liegen kam, wurde der Prozess von Seiten des ULEF «immer entscheidend gefördert und mitgetragen» (Lehrplan der Sekundarschule Basel Stadt, 1990, S. 5). Es übernahm im Rahmen unzähliger Fortbildungsveranstaltungen die Aufgabe, Lehrerinnen und Lehrern wissenschaftliche Erkenntnisse näher zu bringen. Damit hatte das ULEF als kantonales Kompetenz- und Weiterbildungszentrum die Scharnierfunktion zwischen dem wissenschaftlichen Diskurs zur Curriculumtheorie und -forschung einerseits und der praktischen Lehrplanentwicklung andererseits inne.

Guido Harder, der Leiter des ULEF, stand in beruflichem und persönlichem Kontakt mit dem mittlerweile am IPN tätigen Karl Frey. Dies mag erklären, weshalb Frey vor den Lehrpersonen des Kantons Basel-Stadt über «Schwerpunkte der Lehrplanreform für die Sekundarschulen» (Frey, 1974) referierte. In seinen Ausführungen legte Frey auch seine dem Referat vorangegangenen Recherchen offen, wobei er nicht nur auf eine Analyse der bisherigen Lehrplanentwürfe von Basel-Stadt, sondern auch auf zahlreiche Informationsgespräche mit Lehrpersonen, Inspektoren, Rektoren und weiteren Beteiligten Bezug nahm. Freys aufklärendes, orientierendes Engagement in der besagten Lehrplanreform entsprach ganz der Rolle eines externen Beraters oder Tutors, wie sie von den Organisatoren der Reform vorgesehen war (Harder, 1975). Ein Blick in die Jahresberichte und weitere Archivalien der FAL zeugt von unzähligen weiteren Fortbildungs- und Informationsveranstaltungen, bei denen die FAL-Mitglieder als Experten in beratender Rolle auftraten.

Dass die Beratungstätigkeit für Basel nicht singulär war, mögen die fünf folgenden Beispiele verdeutlichen, die auch die Vielfältigkeit dieser Beratungstätigkeit dokumentieren: Erstens wurde im Kanton Zug während mehr als eines Jahres eine Weiterbildungsveranstaltung im Sinne der Unterrichtsberatung durchgeführt. Es standen konkrete Fragen der Lehrpersonen zu Erziehungsstilen oder Unterrichtsvorbereitungen zur Diskussion; daneben wurden Fragen zur Messung des Lernerfolgs oder zur Beurteilung thematisiert (Santini, 1973). Zweitens waren Mitglieder der FAL an den Tagungen des Schweizerischen Pädagogischen Verbandes (Schweizerischer Pädagogischer Verband, 1971; vgl. Hoffmann-Ocon \& Metz, 2013) in Hitzkirch als Kursleiter oder referierend aktiv. Es galt «Informationen über wissenschaftlich begründete Modelle der Unterrichtsvorbereitung» zu vermitteln, die bisherige Praxis einer «kritischen Beobachtung» zu unterziehen oder «Mittel zur Evaluation von Unterricht» zu diskutieren, so die Vorankündigung der Ziele im Tagungsprogramm (Schweizerischer Pädagogischer Verband, 1971). 
Die Erkenntnisse der Curriculumforschung fanden drittens auch ausserhalb der Fortbildung von Lehrerinnen und Lehrern Resonanz. Unter Titeln wie "Warum Schule?» oder «Was lernen unsere Kinder nach dem neuen Lehrplan?» leisteten Joseph Eigenmann und Anton Strittmatter im Kanton Freiburg Öffentlichkeitsarbeit und rahmten so das Referat des damaligen Schulinspektors Schneuwly zur aktuellen Schulreform Freiburgs. Die Veranstaltung empfahl sich «besonders den Eltern, Männern und Frauen, die sich mit der Ausbildung unserer Schuljugend befassen» (Frauenvereinigung FR, 1973).

Die Konzepte der FAL wurden viertens im Bereich der Berufsbildung wahrgenommen. Die Schweizerischen Bundesbahnen (SBB) planten ein neues Ausbildungszentrum und interessierten sich für die Frage, wie die Räumlichkeiten auszugestalten seien, damit sie neusten Erkenntnissen der Bildungswissenschaft entsprechen, und welche Lehr- und Lernmethoden vielversprechend und damit im Einklang wären. Aus dieser Anfrage resultierte ein Konzept der FAL, mit dem die Beziehungen zwischen Unterrichtszielen, -methoden und -medien konzeptuell gefasst und entsprechende Vorschläge formuliert wurden (Eigenmann, 1972). Dass die Entwicklungs- und Beratungstätigkeit gerade auch in diesem Bereich finanziell interessant war, zeigt sich etwa daran, dass Verträge mit der Sandoz AG und der Wander AG abgeschlossen wurden, welche den Verkauf von Curriculumentwürfen und Beratungsleistungen für Ausbildungszentren, die geplant wurden, regelten (Isenegger \& Santini, 1973).

Letztlich waren einzelne Mitglieder der FAL in die Enquête des SWR involviert. Der SWR erhob für die Schweiz Schwerpunkte vordringlicher Forschung und unterbreitete anschliessend dem Bundesrat entsprechende Vorschläge zur Forschungsförderung. Drei der fünf Verfasser des Berichtsteils zur Bildungsforschung gehörten der FAL an (Frey, 1970b). Das starke Engagement der FAL-Mitglieder in diesem Bereich dürfte vor allem zwei Ursachen haben: Einerseits verfügte die FAL aufgrund ihrer bisherigen Umfragen zu Schweizer Lehrplänen über die erforderliche Erfahrung in der Durchführung von Enquêten vergleichbaren Ausmasses. Andererseits hatte Karl Frey selbst Einsitz im Schweizerischen Wissenschaftsrat. Eines der Resultate der Erhebung war letztlich, dass die Curriculumforschung künftig zu den prioritär zu fördernden Bereichen der Bildungsforschung in der Schweiz zählen sollte. Die FAL promovierte also durch ihre Expertentätigkeit auch ihre eigenen Forschungsschwerpunkte.

Die bisher ausgeführten Beispiele zu Beratungsleistungen der FAL stehen allesamt für die ausseruniversitäre Verbreitung der curriculumbasierten Modelle und Konzepte auf unterschiedlichen Ebenen und in unterschiedlichen Bereichen des Bildungssystems. Sie zeugen vom grossen Informationsbedarf und von der grossen Nachfrage nach entsprechend qualifizierter Expertise; beides war letztlich durch die Forderung nach stärkerer Wissenschaftsorientierung mit verursacht worden. Die Freiburger Wissenschaftler waren durch ihre Analysen gleichzeitig Mitproduzenten dieses Bedarfs und bedienten ihn, indem sie Beratungsfunktionen in den laufenden Lehrplanreformen der Kantone, aber auch in vielen 
andern Bereichen übernahmen. Sie vermittelten Informationen sowohl bei konkreten Prozessfragen in der Ausarbeitung neuer Lehrpläne auf Verwaltungsebene als auch für die Unterrichtspraxis der Lehrpersonen. Selbst in der Erwachsenenbildung und in der Privatwirtschaft wurde curriculumspezifische Beratung nachgefragt.

\section{Zwischen Nützlichkeitsversprechen der Erziehungswissenschaft und Beratungsbedarf der Bildungspolitik}

Am Beispiel der wissenschaftlichen und beratenden Tätigkeiten der Freiburger Arbeitsgruppe für Lehrplanforschung im Kontext der Schweizer Curriculumreformen verweisen die Analysen auf einen polymorphen Charakter des Transfers von wissenschaftlichen Inhalten und Konzepten in bildungspolitische Debatten und Prozesse. Transfer kann nicht einfach als blosser Import oder einfache Rezeption von wissenschaftlich propagierten Konzepten zur Konstruktion von Lehrplänen verstanden werden, gerade wenn der Transferbegriff zur Erklärung von Ursachen der Lernzielorientierung in der neuen Lehrplangeneration der 1970er- und 1980er- Jahre herangezogen wird. Seit der Verwendungsforschung der späten 1980er-Jahre (Beck \& Bonss, 1989; Drerup, 1987; König \& Zedler, 1989) ist weitgehend unbestritten, dass der Transfer von wissenschaftlichem Wissen produktiv, konstruktiv und selektiv, also mit Transformationen des Wissens verbunden ist. Es ist deshalb auch auf die Transformationsleistungen der Freiburger Curriculumforscher zu verweisen, welche Elemente der angelsächsischen curriculum studies (Kridel, 2010; Malewski, 2009) durch Adaptionen, aber auch durch Verengungen für die Lehrplanreformen in den Schweizer Kantonen erst anschlussfähig machten. Die FAL etablierte Curriculumfragen als Gegenstand von Forschung, als Ausbildungsinhalt in der Aus- und Weiterbildung von Lehrerinnen und Lehrern, aber auch durch Beratung in Bildungspolitik und -verwaltung. Die Wirksamkeit der FAL lag aber nicht nur in der Transformation von Theorien und Konzepten im Sinne ihrer Adaptation an Bedürfnisse von Bildungsverwaltung und Schulpraxis, sondern auch im Seitenwechsel des Personals und in ihrer Beratungsleistung. Dass die FAL die Lehrplanreformen der 1980er-Jahre in vielen Kantonen stark beeinflusste und wesentlich dafür sorgte, dass curriculares Wissen in Bildungsverwaltung und Schulpraxis transferiert wurde, lag nicht allein an einem dieser Faktoren, sondern an der Gleichzeitigkeit und dem Zusammenspiel aller drei Faktoren, gewissermassen an deren Koinzidenz.

Die Überzeugungen und Absichten der Curriculumforschenden der FAL entwickelten sich im Kontext der Empirisierung, der Fortschrittsorientierung und der Planungsgläubigkeit (Stettler, 1994) während der Bildungsexpansion vor allem der 1960er-Jahre. Sie konnten im Bereich der Lehrplanrevision 
offensichtlich auch aufrechterhalten werden, nachdem die Wirtschaftskrise seit Mitte der 1970er-Jahre eher eine pessimistische Zukunftsstimmung und Planungsskepsis provoziert hatten. Die Idee von rationaleren Grundlagen für bildungspolitische Entscheide war en vogue, weshalb die Curriculumforschung als Alternative zur normativen Tradition der Geisteswissenschaften Nachfrage, aber auch entsprechende finanzielle Unterstützung fand. Der Transfer und die Verbreitung der Konzepte der FAL wurden als legitim, ja notwendig erachtet und entsprechend gefördert. Während die Nachfrage nach Beratung aufgrund der gestiegenen Komplexität der Sachfragen und vor dem Hintergrund des politischen Willens zur Harmonisierung der kantonalen Bildungssysteme über Lehrplanreformen gegeben war, traten die Mitarbeiter der FAL vor allem in den 1970er- und 1980er-Jahren als Experten für die Lösung jener Probleme auf, die sie Ende der 1960er- und zu Beginn der 1970er-Jahre in ihren Analysen überhaupt erst als Probleme identifiziert hatten.

Dass Wissenschaft grundsätzlich aufgerufen ist, einen Beitrag zur Lösung gesellschaftlicher Probleme zu leisten, kam der FAL sehr entgegen. Sie ging auf Nützlichkeitserwartungen einer sich rasch verändernden und expandierenden Bildungsverwaltung geschickt ein, stellte ,objektive', im Sinne von theoretisch fundierten und empirisch geprüften, Konzepte und Lösungen in Aussicht, unterstützte die bildungspolitische Praxis bei deren Entwicklung und Umsetzung, insbesondere im Bereich der Lehrplanrevision, und etablierte sich in Teilbereichen der Bildungsforschung als Beratungsagentur. Dieses Arrangement erlaubte es der FAL, sich in kurzer Zeit als eines der wichtigen ,modernen Bildungsforschungszentren der Schweiz zu positionieren und diese Position während etwa eines Jahrzehnts zu halten. ,Modern' im Sinne von innovativ, aber auch im Sinne von zukunftsweisend war die FAL zumindest in dreierlei Hinsicht: Erstens warb sie durch ihre Entwicklungs- und Beratungstätigkeit eine für damalige Verhältnisse grosse Summe an Drittmitteln ein, was ihren Ausbau und die Kontinuität während einer bestimmten Zeit erst erlaubte. Zweitens kam sie den Nützlichkeitserwartungen der Bildungsverwaltung, wie sie auch heute in Bildungspolitik und Bildungsverwaltung zum Teil vorherrschen, stark entgegen. Drittens lag ihr Erfolg auch darin, dass sie sich als grössere Forschungsgruppe formieren konnte, was für die 1970er-Jahre in der Schweiz im Bildungsbereich noch untypisch war. Zumindest einen Teil ihrer Legitimation bezog die FAL aus ihrem wissenschaftlichen Regress auf amerikanische Forschungsliteratur.

In der beruflichen Weiterentwicklung mancher Mitglieder der FAL zeigt sich ein weiterer Transfermechanismus: Die Nützlichkeitserwartungen von Seiten der Bildungspolitik und Bildungsverwaltung wurden so nicht zuletzt personell eingelöst: Die FAL stellte wissenschaftlich gut qualifiziertes Personal zur Verfügung, dem allerdings zu dieser Zeit wegen des geringen Ausbaus der Pädagogik bzw. Erziehungswissenschaft an den Universitäten (Criblez, 2002) - abgesehen vom Leiter der FAL - eine akademische Laufbahn verwehrt blieb. Die Grenzen zwischen Wissenschaft und Verwaltung, die bis in die 1990er- 
Jahre nicht sehr durchlässig waren (Gretler, 2001), konnten durch den Transfer von Personal zumindest aufgeweicht werden, wie am Beispiel der Zentralschweiz gezeigt werden konnte. In diesem Sinne war die FAL auch ein Netzwerk, das nicht einfach innerakademisch wirksam war, sondern zwischen Wissenschaft, Bildungspolitik bzw. Bildungsverwaltung und Lehreraus- und -weiterbildung aufgespannt wurde.

Letztlich sind die Arbeiten der FAL auch Teil genereller Entwicklungen, die nicht auf die deutschsprachige Schweiz begrenzt waren: etwa der Empirisierung der Pädagogik bzw. des Wandels der Pädagogik zur Erziehungswissenschaft, aber auch Teil eines übergeordneten Prozesses der Verwissenschaftlichung von Politik und Gesellschaft. Jürgen Habermas hatte diesen Prozess 1964 in seinem Aufsatz «Verwissenschaftlichte Politik und öffentliche Meinung» noch so umschrieben: «Die Verwissenschaftlichung der Politik bezeichnet heute noch keinen Tatbestand, aber immerhin eine Tendenz, für die sich Tatbestände zitieren lassen» (S. 54). Am Ende der in diesem Beitrag thematisierten Zeitperiode wurde dieser Prozess nicht mehr als Tendenz, sondern affirmativ beschrieben: «Verwissenschaftlichung der Gesellschaft - Politisierung der Wissenschaft» (Weingart, 1983). Die FAL war in der Bildungsforschung der Schweiz ein wichtiger Akteur in diesem Prozess. Inwiefern ihre Aktivitäten mitunter auch von Wissenschaft in Politik umschlugen und es gerade dadurch zur «Politisierung der Wissenschaft» kam, wäre in weiteren Analysen zu prüfen.

\section{Anmerkungen}

1 Die Debatten der 1970er-Jahre rund um Lernzieltaxonomien, um die kantonalen Harmonisierungsbestrebungen via Lehrpläne, aber auch um die übergeordnete Frage, welches schulische Wissen wie in Lehrplänen festgehalten sein soll, sind nicht singulär. Vielmehr bestimmten die Kernprobleme der Curriculumdiskussion (zu nennen sind insbesondere das Legitimations-, das Deduktions-, das Taxonomie- und das Operationalisierungsproblem) unter anderen Vorzeichen auch die Diskussionen um Bildungsstandards und Lehrplan 21.

2 Unsere Aussagen beruhen einerseits auf der Analyse der Archivalien der Freiburger Arbeitsgruppe, die bei der Schweizerischen Koordinationsstelle für Bildungsforschung $(\mathrm{SKBF})$ in Aarau aufbewahrt werden. Andererseits beziehen wir uns auf die Monografien und Zeitschriftartikel der FAL-Mitglieder, welche sowohl während als auch nach ihrer Tätigkeit bei der Arbeitsgruppe entstanden sind. Ergänzend wurden Experteninterviews mit ehemaligen Mitgliedern der FAL geführt.

3 Nach seinem Studium der Psychologie und Theologie in Leuven, Münster und Freiburg promovierte Karl Frey (1942-2005) an der Universität Freiburg in Pädagogik und gründete da 1968 die Freiburger Arbeitsgruppe für Lehrplanforschung am Pädagogischen Institut. 1971 folgte er dem Ruf auf eine ordentliche Professur nach Kiel und leitete dort das Institut für Pädagogik der Naturwissenschaften. Seit 1988 versah er die Professur für Erziehungswissenschaft an der Eidgenössischen Technischen Hochschule (ETH) in Zürich.

4 Der vorliegende Forschungsstand zur Lehrplanarbeit in den 1970er-Jahren bezieht sich ausschliesslich auf die Schweizer Situation. Generell ist die historisch-analytische Literatur zur Curriculumdebatte der 1960er-/1970er-Jahre für den deutschsprachigen Raum spärlich. Weiterführend: Haft \& Hopmann (1987); Hopmann (1988); Künzli (1988); 
Horlacher \& De Vincenti (2014); Künzli \& Hopmann (1998). Nicht zuletzt aufgrund einer deutlich breiteren Auslegung des Curriculumbegriffes in den angelsächsischen curriculum studies als im deutschen Sprachraum und entsprechend zahlreichen Publikationen wird an dieser Stelle auf eine Darstellung des angelsächsischen Forschungsstandes verzichtet.

5 Die Freiburger Arbeitsgruppe für Lehrplanforschung zählte während ihrer Aktivzeit von 1968 bis 1978 insgesamt 20 Mitarbeiter, wobei je nach Anzahl laufender Projekte knapp zehn gleichzeitig angestellt waren. Während sich zum Beispiel Joe Brunner und Joseph Eigenmann acht Jahre bei der FAL engagierten, zählen Urs Isenegger, Kurt Aregger, Kurt Bosshard oder Ralph Horn zu den Mitarbeitern, die die FAL in den frühen 1970er-Jahren bereits wieder verliessen. Ihnen folgten Kurt Schmid, Ernst Preisig, Daniel Huber oder Beat Meyer nach. Sie waren bis 1977 bzw. 1978 in der FAL aktiv. Für das Bestehen der FAL ist weder ein genaues Gründungsdatum noch ein offizielles Datum der Auflösung bekannt. Im letzten Jahresbericht von 1978 werden finanzielle Schwierigkeiten als Gründe für die Auflösung geltend gemacht (Eigenmann, 1978). Im Laufe des knapp zehnjährigen Bestehens der FAL wandelte sich ihre inhaltliche Ausrichtung von empirischen Vorarbeiten der Gründerjahre über eine erste Phase der Curriculumforschung hin zu didaktischen und wissenschaftstheoretischen Fragen wie auch Untersuchungen zur Wirksamkeit von Unterricht (Gretler, 1979).

6 Dies veranlasste Frey auch dazu, in seinen Anmerkungen weiterführende Präzisierungen vorzunehmen: "Curriculum» und «Lehrplan» seien prinzipiell synonym zu betrachten, wobei erst die letzten Jahre zu einer Wiederentdeckung des Curriculumbegriffes im deutschen Sprachraum geführt hätten, was wiederum der Curriculumforschung geschuldet sei (Frey, 1969b).

7 Diese Reform der Sekundarstufe I war in der Zentralschweiz einerseits durch die international geführte Gesamtschuldiskussion, andererseits durch eine Vorgabe des Schulkonkordates von 1970 bedingt: Der obligatorische Unterricht sollte in Zukunft für alle Schülerinnen und Schüler schweizweit neun Jahre dauern. In einzelnen Kantonen bestand damals lediglich eine obligatorische Unterrichtspflicht von sieben, zum Teil von acht Jahren (Manz, 2011).

8 Die Fortführung der Aufbauarbeiten des ZBS übernahm 1975 ein weiteres FAL-Mitglied, Anton Strittmatter, der bis 1987 als dessen Leiter amtete. Auch Xaver Winiger engagierte sich als ehemaliges FAL-Mitglied ab 1976 beim ZBS.

9 Demnach erfordert ein neues Curriculum eine neue Art der Unterrichtsvorbereitung und -gestaltung und stellt die Lehrpersonen vor neue Herausforderungen und Probleme. Die Lehrerschaft würde aber, so die These des Implementationsansatzes, mit länger andauernder Partizipation am Reformprozess innovationsbereiter.

10 Mit Urs Peter Lattmann (bis September 1971) und Anton Strittmatter (seit September 1971) amteten zwei FAL-Mitglieder als wissenschaftliche Sekretäre, Urs Isenegger, Ko-Leiter der FAL, ersetzte im Juni 1971 Karl Frey als Mitglied der LEMO-Kommission (Müller et al., 1975, S. 347f.).

11 Rückblickend muss denn die Absicht der kantonalen Erziehungsdirektoren, in den 1970erJahren die Schweizer Lehrerinnen- und Lehrerbildung zu koordinieren, als gescheitert betrachtet werden (Criblez, 1999).

\section{Bibliographie}

Aregger, K. (1971a). Lehrplanreform als Grundlage der Schulreform. Schweizerische Lehrerzeitung, (50), 1824-1831.

Aregger, K. (1971b). Implementation im lehrergesteuerten Unterricht. In K. Aregger \& U. Isenegger (Hrsg.), Curriculumprozess: Beiträge zur Curriculumkonstruktion und-implementation (S. 174-188). Basel: Beltz. 
Aregger, K. (1975). Organisation der Curriculumentwicklung. Skizzen aus der Organisationsforschung. Kiel: Institut für die Pädagogik der Naturwissenschaften.

Aregger, K., Brunner, J. \& Isenegger, U. (Hrsg.) (1972). Lehrplanreform als Grundlage der Schulreform. Freiburg: Kantonaler Lehrmittelverlag.

Aregger, K. \& Frey, K. (1975). Ein Modell zur Integration von Theorie und Praxis in Curriculum-Projekten: Das Generative Leitsystem. Kiel: Institut für die Pädagogik der Naturwissenschaften.

Ash, M. G. (2006). Wissens- und Wissenschaftstransfer. Berichte zur Wissenschaftsgeschichte, 29, (3), 181-189.

Ausubel, D. P. (1968). The psychology of meaningful verbal learning: An introduction to school learning. New York: Grune \& Stratton.

Bain, D., Brun, J., Hexel, D. \& Weiss, J. (Hrsg.) (2001). Die Geschichte der Bildungsforschungsstellen in der Schweiz 1960-2000. Neuchâtel: IRDP.

Beck, U. \& Bonss, W. (Hrsg.). (1989). Weder Sozialtechnologie noch Aufklärung? Analysen zur Verwendung wissenschaftlichen Wissens. Frankfurt/M.: Suhrkamp.

Bloom, B. S. et al. (1956/1972). Taxonomie von Lernzielen im kognitiven Bereich. Weinheim: Beltz.

Bloom, B. S. \& Krathwohl, D. R. (1964). Taxonomy of educational objectives: The classification of educational goals. New York: Longman.

Bosche, A. (2013). Schulreformen steuern: Die Einführung neuer Lehrmittel und Schulfächer an der Volksschule (Kanton Zürich, 1960er-bis 1980er-Jahre). Bern: hep.

Bosche, A. (2016). The back office of school reform: Educational planning units in Germanspeaking Switzerland (1960s and 1970s). Paedagogica Historica, 52, (4), 380-394.

Bruner, J. (1966). Toward a theory of instruction (5. Aufl.). Cambridge: Belknap.

Criblez, L. (1999). Neue Schwerpunkte für die Lehrerbildungspolitik: Einige Konsequenzen aus dem Nationalfondsprojekt zur "Wirksamkeit der Lehrerbildungssysteme» für die künftige Lehrerinnen-und Lehrerbildung. Beiträge zur Lehrerbildung, 17, (2), 162-173.

Criblez, L. (2002). Fragil und unstet - Zur Entwicklung und aktuellen Situation der Erziehungswissenschaften an den Universitäten der deutschsprachigen Schweiz. In R. Hofstetter $\&$ B. Schneuwly (Hrsg.), Science(s) de l'éducation 19e-20e siècle. Entre champs professionnels et champs disciplinaires (S. 425-453). Bern: Lang.

Criblez, L. (2008). Vom strikten Bildungsföderalismus zur bescheidenen Schulkoordination. In L. Criblez (Hrsg.), Bildungsraum Schweiz-Historische Entwicklung und aktuelle Herausforderungen (S. 251-276). Bern: Haupt.

Criblez, L. (2010). Die Reform der Lehrerinnen- und Lehrerbildung in der Schweiz seit 1990: Reformprozesse, erste Bilanz und Desiderata. In H. Ambühl \& W. Stadelmann (Hrsg.), Tertiarisierung der Lehrerinnen- und Lehrerbildung: Bilanz und Perspektiven (S. 11-47). Bern: EDK.

Criblez, L. (2012). Die Expansion der Bildungsverwaltung in den 1960er und 1970er Jahren - am Beispiel der Kantone Zürich und Bern. In M. Geiss \& A. De Vincenti (Hrsg.), Verwaltete Schule (S. 109-129). Wiesbaden: VS Verlag für Sozialwissenschaften.

Criblez, L., Imlig, F., Montanaro-Batliner, I., Ruoss, T. \& Völgyi, M. (2013). Von der Aargauer Lehramtsschule zur Intensivweiterbildung 1973-2013. Baden: hier+jetzt.

Di Maggio, P. J. \& Powell, W. W. (1983/2009). Das «stahlharte Gehäuse» neu betrachtet: Institutionelle Isomorphie und kollektive Rationalität in organisationalen Feldern. In S. Koch \& M. Schemmann (Hrsg.), Neo-Institutionalismus in der Erziehungswissenschaft (S. 57-84). Wiesbaden: VS Verlag für Sozialwissenschaften.

Drerup, H. (1987). Wissenschaftliche Erkenntnis und gesellschaftliche Praxis. Anwendungsprobleme der Erziehungswissenschaft in unterschiedlichen Praxisfeldern. Weinheim: Deutscher Studien Verlag.

Dunkel, S. (2014). Institutionalisierte Lehrer- und Lehrerinnenfortbildung im Kanton BaselStadt. Rekonstruktion der Gründungs- und Institutionalisierungsgeschichte des Instituts für 
Unterrichtsentwicklung und Lehrer- und Lehrerinnenfortbildung (1900-1970). Unveröffentlichte Lizenziatsarbeit am Institut für Erziehungswissenschaft der Universität Zürich.

Eigenmann, J. (1972). Konzept für ein Ausbildungszentrum der SBB. FAL-Archiv. Aarau: SKBF.

Eigenmann, J. (1978). Tätigkeitsbericht FAL 1978. Freiburg: Forschungszentrum - FAL; Pädagogisches Institut. FAL-Archiv. Aarau: SKBF.

Eigenmann, J. \& Schmid, K. (1978). Zwei Schwerpunkte aktueller Curriculumdiskussion. In W.-R. Minsel (Hrsg.), Curriculum und Lehrplan (S. 168-187). München: Urban \& Schwarzenberg.

Frauenvereinigung FR (1973). Einladung zum Orientierungsabend. FAL-Archiv. Aarau: SKBF. Frey, K. (1968a). Der Bildungsauftrag der Realschule (2. Aufl.). Weinheim: Beltz.

Frey, K. (1968b). Der Lehrplan der Real-, Sekundar- und Bezirksschulen: Eine vergleichende Analyse zur Koordination und Neugestaltung der kantonalen Lehrpläne in der Schweiz. Weinheim: Beltz.

Frey, K. (1969a). Der Ausbildungsgang der Lehrer: Eine Modellanalyse des Unterrichts in den 52 Lehrerbildungsanstalten zum Zwecke der Curriculumreform. Weinheim: Beltz.

Frey, K. (1969b). Lehrplananalyse als Teil der Curriculumforschung. International Review of Education, 15, (1), 4-26.

Frey, K. (1970a). Die Taxonomien und das Curriculum. Paedagogica Europaea, 6, 50-72.

Frey, K. (1970b). Vorgehen für die Erstellung des Berichtes «Bildungsforschung" des Schweizerischen Wissenschaftsrates. FAL-Archiv. Aarau: SKBF.

Frey, K. (1971). Theorien des Curriculums. Weinheim: Beltz.

Frey, K. (1974). Schwerpunkte der Lehrplanreform für die Sekundarschulen. FAL-Archiv. Aarau: SKBF.

Frey, K., Lattmann, U. P. \& Aebli, H. (1971). Kriterien in der Curriculumkonstruktion. Weinheim: Beltz.

Gagné, R. M. (1965/1969). Die Bedingungen des menschlichen Lernens (Orig: The Conditions of Learning). Hannover: Schrödel.

Göldi, S. (2011). Von der bloomschen Taxonomy zu aktuellen Bildungsstandards: zur Entstehungs- und Rezeptionsgeschichte eines pädagogischen Bestsellers. Bern: hep.

Goodlad, J. I. (1964). School curriculum reform in the United States. New York: Fund for the Advancement of Education.

Gretler, A. (1979). Löst sich die Freiburger Arbeitsgruppe für Lehrplanforschung (FAL) auf? Bildungsforschung und Bildungspraxis, 1, (1), 95-97.

Gretler, A. (2001). Entwicklungen der universitären und ausseruniversitären Bildungsforschung - Gewichtsverschiebungen in der zweiten Hälfte des 20. Jahrhunderts. In D. Bain, J. Brun, D. Hexel \& J. Weiss (Hrsg.), Die Geschichte der Bildungsforschungsstellen in der Schweiz 1960-2000 (S. 311-323). Neuchâtel: IRDP.

Guilford, J. P. (1959/1964). Persönlichkeit. Logik, Methodik und Ergebnisse ihrer quantitativen Erforschung (Orig. Personality). Weinheim: Beltz.

Habermas, J. (1964). Verwissenschaftlichte Politik und öffentliche Meinung. In R. Reich (Hrsg.), Humanität und politische Verantwortung (S. 54-73). Erlenbach-Zürich: Rentsch.

Haft, H. \& Hopmann, S. (1987). Lehrplanarbeit in der Bundesrepublik Deutschland. Die deutsche Schule, 79, (4), 508-518.

Hameyer, U. (1983). Systematisierung von Curriculumtheorien. In U. Hameyer, K. Frey \& H. Haft (Hrsg.), Handbuch der Curriculumforschung (S. 54-100). Weinheim/Basel: Beltz.

Harder, G. (1975). Jahresbericht. Basel: Institut für Unterrichtsfragen und Lehrerfortbildung (ULEF).

Hoffmann-Ocon, A. \& Metz, P. (2013). Nähe und Distanz. Zur Geschichte und zum Verhältnis des Gymnasial- und des Seminarlehrervereins. Gymnasium Helveticum, 67, (5), 13-19. 
Hopmann, S. (1988). Lehrplanarbeit als Verwaltungshandeln. Kiel: Institut für die Pädagogik der Naturwissenschaften.

Horlacher, R. \& De Vincenti, A. (2014). From rationalist autonomy to scientific empiricism: A history of curriculum in Switzerland. In W. F. Pinar (Ed.), International handbook of curriculum research (S. 476-492). New York: Routledge.

Horn, R., Morger, S., Regli, K. \& Zumthrum, J. (1969). Ausbildung und Anstellung der Gymnasiallehrer: eine vergleichende Dokumentation als Grundlage für die Reformplanung. Freiburg: Pädagogisches Institut Universität Freiburg.

Isenegger, U. (1972). Jahresbericht 1971 zuhanden der Generalversammlung der FAL. Freiburg: Forschungszentrum FAL; Pädagogisches Institut. FAL-Archiv. Aarau: SKBF.

Isenegger, U. (1975). Einleitung. In U. Isenegger \& B. Santini (Hrsg.), Begriff und Funktionen des Curriculums (S. 10-19). Weinheim: Beltz.

Isenegger, U. \& Santini, B. (1973). Tätigkeitsbericht FAL 1972. Freiburg: Forschungszentrum FAL; Pädagogisches Institut. FAL-Archiv. Aarau: SKBF.

Jenzer, C. (1983). Gesamtschule Dulliken 1970-1980. Idee, Realisierung, Resultate, Ausblick. Bern: Haupt.

Jenzer, C., Weiss, J. \& Strittmatter, A. (Hrsg.). (1978). Schulkoordination über Lehrplanreform. Frauenfeld: Huber.

Joyeux-Prunel, B. (2002). Les transferts culturels. Hypothèses, 6, (1), 149-162.

Kliebard, H. M. (1987). The question of Dewey's impact on curriculum practice. Teachers College Record, 89, (1), 139-142.

König, E. \& Zedler, P. (Hrsg.). (1989). Rezeption und Verwendung erziehungswissenschaftlichen Wissens in pädagogischen Handlungs- und Entscheidungsfeldern. Weinheim: Deutscher Studien Verlag.

Kridel, C. A. (Ed.). (2010). Encyclopedia of curriculum studies. Thousand Oaks, CA: SAGE.

Künzli, R. (1988). Lehrplanung - Der "unmögliche Diskurs» oder die Sicherung von Permanenz. In S. Hopmann (Hrsg.), Zugänge zur Geschichte staatlicher Lehrplanarbeit (S. 235-258). Kiel: Institut für die Pädagogik der Naturwissenschaften.

Künzli, R. (2002). Standardisierung der Ausbildung oder ihrer Absolventen? Beiträge zur Lehrerbildung, 20, (1), 21-30.

Künzli, R. (2002/2006). Kantonale Lehrplanpolitik in der Schweiz. In L. Criblez, P. Gautschi, P. Hirt-Monico \& H. Messner (Hrsg.), Lehrpläne und Bildungsstandards: Was Schülerinnen und Schüler lernen sollen (S. 61-78). Bern: hep (Erstveröffentlichung: 2002).

Künzli, R. \& Hopmann, S. (1998). Entscheidungsfelder der Lehrplanarbeit. Grundzüge einer Theorie der Lehrplanung. In R. Künzli \& S. Hopmann (Hrsg.), Lehrpläne: wie sie entwickelt werden und was von ihnen erwartet wird. Forschungsstand, Zugänge und Ergebnisse aus der Schweiz und der Bundesrepublik Deutschland (S. 17-34). Chur: Rüegger.

Kussau, J. \& Oertel, L. (2001). Bildungsexpansion, Reform der Sekundarstufe I und Pädagogische Arbeitsstellen. Schweizerische Zeitschrift für Bildungswissenschaften, 23, (1), 137-163.

Lattmann, U. P. (1976). Reformen in der Lehrerbildung. In E. Egger \& B. Kehrli (Hrsg.), Schulreformen in der Schweiz (S. 191-209). Frauenfeld: Huber.

Lehrplan der Sekundarschule Basel Stadt (1990). Basel: Erziehungsdirektion.

Malewski, E. (Ed.) (2009). Curriculum studies handbook - The next moment. New York: Routledge.

Manz, K. (2011). «Schulkoordination ja - aber nicht so!» Die Anfänge der schweizerischen Schulkoordination (1960-1985). Bern: hep.

Metz, P. (2000). Bildungspolitik und Lehrerbildung: Historische Problemlagen und ihre Lösungen im Aargau. In L. Criblez, R. Hofstetter \& D. Périsset Bagnoud (Hrsg.), La formation des enseignant(e)s primaires - Histoire et réformes actuelles (S. 75-102). Genf/ Bern: Lang.

Miles, M. B. (1961). Innovation in education. New York: Teachers College Press. 
Müller, F. et al. (1975). Lehrerbildung von morgen: Grundlagen - Strukturen - Inhalte. Bericht der Expertenkommission "Lehrerbildung von morgen" im Auftrag der Schweizerischen Konferenz der kantonalen Erziehungsdirektoren. Hitzkirch: Comenius-Verlag.

Nicklas, H. W. (1972). Probleme der Curriculumentwicklung. In W. Klafki \& S. Aust (Hrsg.), Probleme der Curriculumentwicklung (S. 11-22). Frankfurt/M.: Diesterweg.

Realschule Basel Stadt (1975). Lernziele und Stoffpläne der Realschule - Korrekturen - Verlängerungen des Provisoriums. Basel: Erziehungsdepartement.

Rickenbacher, I. (1976). Bildungsplanung und Schulreform in der Zentralschweiz. In K. Widmer (Hrsg.), Bildungsplanung und Schulreform (S. 253-264). Frauenfeld: Huber.

Ringelband, U. (2016). 50 Jahre IPN - Forschen für die Gesellschaft. Kiel: nndruck. Zugriff am 27.07.2017 unter https://www.ipn.uni-kiel.de/de/das-ipn/50-jahre-ipn/IPN_50Jahre_ Broschuere_web.pdf

Robinsohn, S. B. (1967). Bildungsreform als Revision des Curriculum. Neuwied: Luchterhand.

Roth, H. (1963). Die realistische Wendung in der pädagogischen Forschung. Die Deutsche Schule, 55, (3), 109-119.

Rothen, C. (2016a). Educational research within the administration: A booming business in the French-speaking part of Switzerland (1950-1980). Paedagogica Historica, 1-13.

Rothen, C. (2016b). Zwischen Innovation und Administration. Genese der wissenschaftlich orientierten Bildungsplanung in Zürich, Bern und Neuenburg, 1960-1990. In L. Criblez, C. Rothen \& T. Ruoss (Hrsg.), Staatlichkeit in der Schweiz (S. 297-315). Zürich: Chronos.

Santini, B. (1973). Unterrichtsberatung: Vorgehen für das Schuljahr 1973/1974. Freiburg: Forschungszentrum FAL; Pädagogisches Institut. FAL-Archiv. Aarau: SKBF.

Santini, B. (1983). Taxonomien. In K. Frey, U. Hameyer \& H. Haft (Hrsg.), Handbuch der Curriculumforschung (S. 617-641). Weinheim: Beltz.

Schweizerischer Pädagogischer Verband (1971). Programm der SPV Tagung zur Unterrichtsvorbereitung. FAL-Archiv. Aarau: SKBF.

Schweizerischer Wissenschaftsrat (1973). Forschungsbericht. Bd. 1. Bern: SWR.

Stettler, N. (1994). «Die Zukunft ist errechenbar ...». In D. Blanc \& C. Luchsinger (Hrsg.), Achtung: die 50er Jahre! (S. 95-117). Zürich: Chronos.

Strittmatter, A. (1977). ZBS Grundlagen und Tätigkeitsbericht 1974-1976. Luzern: Zentralschweizerischer Beratungsdienst für Schulfragen.

Strittmatter, A. (2003). Hat LEMO den Rahmenlehrplan verpasst? Beiträge zur Lehrerbildung, 21, (3), 334-341.

Taba, H. (1962). Curriculum development. New York: Harcourt, Brace \& World.

Tyler, R. W. (1949). Basic principles of curriculum and instruction. Chicago: The University of Chicago Press.

Weingart, P. (1983). Verwissenschaftlichung der Gesellschaft - Politisierung der Wissenschaft. Zeitschrift für Soziologie 12, (3), 225-241.

Winiger, X. (2007). 32 Jahre pädagogische Stabsstelle Zentralschweiz. Mittendrin, (1), 4-5.

Schlagworte: Lehrplan, Curriculumtheorie, Schulreform, Verwissenschaftlichung, Bildungsverwaltung, Aus- und Weiterbildung von Lehrpersonen 


\section{Transfert du savoir dans le contexte des discussions des années 1970 sur les plans d'études en Suisse}

\section{Ré sumé}

Dans le contexte des discussions menées en Suisse dans les années 1970 sur les plans d'études, cet article examine trois mécanismes centraux de transfert à l'exemple du Freiburger Arbeitsgruppe für Lehrplanforschung (FAL): le transfert des résultats scientifiques américains dans le contexte de réforme curriculaire en Suisse, le transfert du savoir scientifique en matière de changements du personnel de l'Université à la pratique éducative et politique de formation ainsi que le transfert du savoir à travers de consultation politique. Les stratégies et motivations des acteurs impliqués mises en lumière et les processus de réforme abordés démontrent de manière générale que le groupe de travail FAL a apporté une contribution précieuse à la scientifisation des travaux sur les plans d'études dans la Suisse des années 1970.

Mots-clés: Plan d'études, réforme scolaire, scientifisation, administration de l'éducation, formation des enseignants

\section{Trasferimento di saperi nel contesto della discussione svizzera sui curricoli degli anni 1970}

\section{Riassunto}

Il contributo analizza tre meccanismi di trasferimento che hanno caratterizzato, in Svizzera, la discussione sui curricoli degli anni 1970, e che si possono esemplificare attraverso il caso della Freiburger Arbeitsgruppe für Lerhplanforschung (FAL): il trasferimento dei risultati di ricerche statunitensi verso il contesto delle riforme curricolari in Svizzera, il trasferimento di conoscenze scientifichecirca le carriere del personale accademico che dall'università entra nelle scuole pubbliche, e il trasferimento di saperi attraverso consulenze in ambito politico. Lo studio delle strategie e delle ragioni di fondo degli attori coinvolti e la presa in considerazione dei processi di riforma, mostrando che la FAL ha contribuito in maniera importante alla scientificizzazione all'elaborazione della riforma curricolare nella Svizzera degli anni 1970.

Parole-chiave: Piani di studio, Teoria del curricolo, riforma scolastico, scientificizzazione, amministrazione del sistema educativo, formazione dei docenti, formazione continua dei docenti 


\section{Knowledge Transfer in Swiss Curriculum Discussions in the 1970s}

\section{Abstract}

This contribution analyses three central transfer mechanisms in the context of the discussion on the curriculums that took place in Switzerland during the 1970s, using the example of the Freiburger Arbeitsgruppe fü Lehrplanforschung (FAL): the transfer of American research results into Swiss contexts of curriculum reform, the transfer of scientific knowledge through the change of sides of the scientists from the university to jobs in the field of school education and into education politics, as well as the knowledge transfer through political consulting. Strategies and motives of the actors involved will be highlighted and processes of reform analyzed. This will demonstrate, that the FAL had a substantial part in the scientification of the development of curriculums in Switzerland in the 1970 s.

Keywords: Curriculum, curriculum studies, school reform, scientification, educational administration, teacher-training 
